\title{
Advances in radiotherapy for esophageal cancer
}

\author{
Wei Deng ${ }^{1}$, Steven H. Lin ${ }^{2}$ \\ ${ }^{1}$ Department of Radiation Oncology, National Cancer Center/Cancer Hospital, Chinese Academy of Medical Sciences and Peking Union Medical \\ College, Beijing 100021, China; ${ }^{2}$ Department of Radiation Oncology, The University of Texas MD Anderson Cancer Center, Houston, TX, USA \\ Contributions: (I) Conception and design: All authors; (II) Administrative support: SH Lin; (III) Provision of study materials or patients: All authors; \\ (IV) Collection and assembly of data: W Deng; (V) Data analysis and interpretation: All authors; (VI) Manuscript writing: All authors; (VII) Final \\ approval of manuscript: All authors. \\ Correspondence to: Steven H. Lin, MD, PhD. Department of Radiation Oncology, The University of Texas MD Anderson Cancer Center, 1515 \\ Holcombe Blvd., Unit 097, Houston, TX 77030, USA. Email: shlin@mdanderson.org.
}

\begin{abstract}
Esophageal cancer is a common type of malignancy worldwide and usually requires multidisciplinary care. Radiotherapy plays an important part in management of the disease. During the past few years, researchers have made much progress about radiotherapy for esophageal cancer, which was revealed in every aspect of clinical practice. Neoadjuvant chemoradiotherapy remains the standard treatment for locally advanced esophageal cancer, whereas neoadjuvant chemotherapy appears to show less toxicities and non-inferior prognosis. What's more, definitive chemoradiotherapy could be an option for non-surgical candidates and good responders to chemoradiotherapy. Advances in radiation techniques result in higher conformity, homogeneity, more normal tissue sparing and less treatment time. Promising prognoses and less toxicities were also seen in advanced techniques. As radiation dose higher than 50 Gy obtains better local control and survival, simultaneously integrated boost is designed to increase primary tumor dosage and keep prophylactic dose to subclinical areas. Elective nodal irradiation brings about better local control but do not show advantages in survival compared with involved field irradiation (IFI). As a trend, more tolerable chemoradiotherapy regimen would be taken into account in dealing with elderly patients.
\end{abstract}

Keywords: Radiotherapy; esophageal cancer; neoadjuvant chemoradiotherapy; radiation techniques; simultaneously integrated boost; target volume

Submitted Sep 18, 2017. Accepted for publication Nov 07, 2017.

doi: $10.21037 /$ atm.2017.11.28

View this article at: http://dx.doi.org/10.21037/atm.2017.11.28

\section{Introduction}

Esophageal cancer is the eighth most common cancer worldwide, it was estimated 455,800 new cases and over 400,000 deaths annually (1). Esophageal squamous cell carcinoma accounts for most of the histopathological types in Asia, whereas adenocarcinoma is the prevalent subtype in the United States and Western Europe. Radiotherapy acts as an indispensable role in management of esophageal cancer. Recent advances have been made about radiotherapy as to achieve better balance between increasing treatment efficacy and reducing toxicities. This review mainly focuses on the advances in treatment modalities, radiation techniques, radiation dose, target volume and radiotherapy in elderly patients.

\section{Treatment modalities}

\section{Neoadjuvant therapy}

Neoadjuvant chemoradiotherapy remains to be the standard treatment modality for locally advanced esophageal cancer based on meta-analyses and CROSS trial (2-4). In CROSS trial, neoadjuvant chemoradiotherapy significantly increase R0 resection rates $(92 \%$ vs. $69 \%, \mathrm{P}<0.001)$ and improve median overall survival (48.6 vs. 24.0 months, $\mathrm{P}=0.003$ ) (5). Since MAGIC trial verified the efficacy of perioperative chemotherapy in lower esophageal adenocarcinoma and gastroesophageal junction cancer (6), perioperative or preoperative chemotherapy have also been applied in esophageal adenocarcinoma. It remains uncertain that 
which one outperforms the other. A randomized trial from Karolinska University Hospital compared the efficacy of neoadjuvant chemotherapy with or without radiotherapy in esophageal or gastroesophageal junction cancer patients (7). Neoadjuvant chemoradiotherapy turned out to have higher pathologic complete response (pCR) rate (28\% vs. $9 \%$, $\mathrm{P}=0.002), \mathrm{R} 0$ resection rate $(87 \%$ vs. $74 \%, \mathrm{P}=0.04)$, and lower lymph node metastases rate (35\% vs. $62 \%, \mathrm{P}=0.001)$, while the overall survival rate was not significantly improved ( $47 \%$ vs. 49\%, $\mathrm{P}=0.77$ ). Notably, the neoadjuvant chemoradiotherapy group have more severe postoperative complications according to Clavien-Dindo system (8) and cause more deaths that are unrelated to disease progression. Nevertheless, none significant difference of mortality was observed among patients with neoadjuvant chemoradiotherapy in other trials and meta-analysis (9-11). What's more, the benefit for the overall survival among patients with neoadjuvant chemoradiotherapy was observed by Stahl et al. (12) (3 year overall survival $47.4 \%$ vs. $27.7 \%$, HR 0.67, 95\% CI: 0.41-1.07, P=0.07) and Swisher et al. (13) (3 year overall survival $48 \%$ vs. $29 \%, \mathrm{P}=0.04$ ), although these two studies were criticized for the low radiation dose (30 Gy) and none intention-to-treat analysis. Nowadays, it remains controversial whether the toxicities of neoadjuvant chemoradiotherapy will convert into increased postoperative complications or cause negative effect on survival (Table 1). The potential prognostic factors like stage, histology type, radiation field, proficiency of surgeon and postoperative supportive care may also play vital role in prognosis and the treatment choice.

\section{Definitive chemoradiotherapy}

Definitive chemoradiotherapy is reserved for certain subgroups of patients, especially in those with cT4b, extensive lymph node metastasis or not unsuitable for surgery. It had been proved to be well tolerated with comparable efficacies to surgery. In a phase II trial, chemoradiotherapy with docetaxel and cisplatin improved the median overall survival time to 23 months among patients (17). Also, compared with traditional cisplatin and fluorouracil, carboplatin and paclitaxel resulted in similar survival but lower toxicities and higher treatment compliance (18). Paclitaxel has been widely used in respect of definitive chemoradiotherapy.

For patients who meet surgery contraindications or show good response to chemoradiotherapy, definitive chemoradiotherapy may not be inferior to trimodality surgery. In FFCD 9102 trial, patients with T3N0-1 esophageal cancer (mostly squamous cell carcinomas) were randomized to surgery or definitive chemoradiotherapy. The 2 -year overall survival rates were $34 \%$ and $40 \%$, median overall survival were 17.7 months and 19.3 months $(\mathrm{P}=0.44)$, respectively. Patients received surgery had higher local control rates $(66.4 \%$ vs. $57.0 \%, \mathrm{P}=0.0014)$ (19). Stahl et al. obtained similar results among patients with esophageal squamous cell carcinoma. Compared to surgery, definitive chemoradiotherapy had an equivalent 2-year overall survival rate $(39.9 \%$ vs. $35.4 \%)$, relatively decreased 2-year local progression free survival rate $(64.3 \%$ vs. $40.7 \%$, $\mathrm{P}=0.003)$ and lower treatment mortality rate compared with surgery (12.8\% vs. $3.5 \%, \mathrm{P}=0.03)$ (20). A case-control study shows that patients with complete clinical response (cCR) had a better prognosis after chemoradiotherapy compared with surgery (median survival time, 31 vs. 83 months, $\mathrm{P}=0.001$ ). Recurrences tend to occur in locoregional sites, which indicated the probability of intervention for salvage surgery (21). Several studies have shown that salvage surgery after chemoradiotherapy achieve comparable survival rates (3-year overall survival 43-48\%) with planned surgery $(22,23)$. Considering patients with squamous cell carcinomas are more likely to have locoregional recurrences (24) and most of the patients aforementioned are squamous cell carcinomas, definitive chemoradiotherapy and salvage surgery should be taken as the first-line treatment, especially among responders to chemoradiotherapy. While persistent tumor after chemoradiotherapy tends to be more aggressive and have a poorer survival.

\section{Radiation techniques}

\section{Intensity modulated radiotherapy}

Three-dimensional conformal radiotherapy (3DCRT) remains the standard radiation technique for esophageal cancer. Intensity-modulated radiotherapy (IMRT) with inverse planning could produce more conformal dose distribution to the target and steeper dose gradients around the target edges than 3DCRT, which involves forward planning with manually modifying beam parameters. This capability makes it possible to give a high dose to the target volume while sparing adjacent normal tissues. Surrounded by bilateral lungs and mediastinal organs, esophageal cancer irradiation usually causes dose impacts on lungs and heart, which may result in acute toxicities dominated by radiation pneumonitis and late toxicities such as cardiac events, 


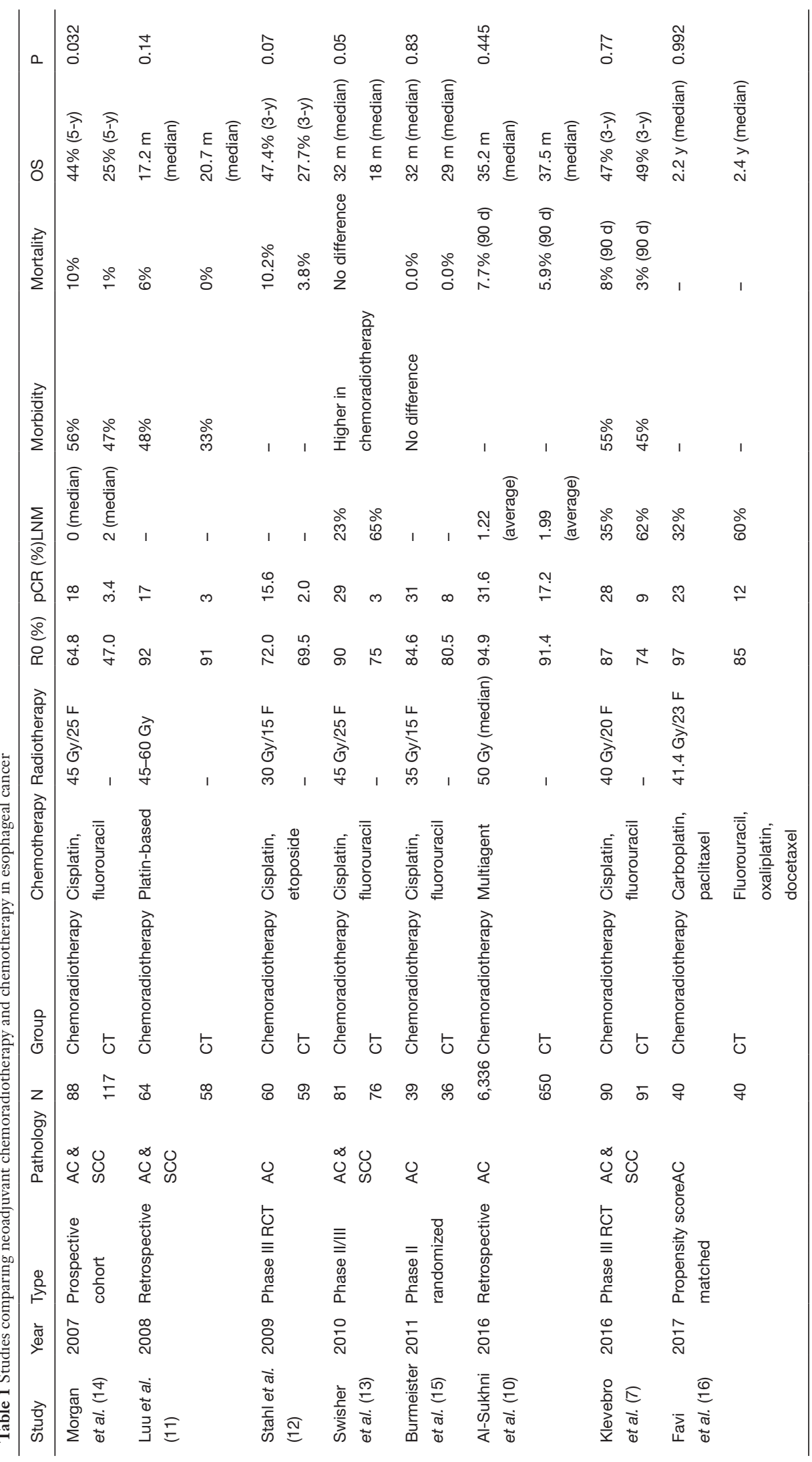


pulmonary fibrosis, or deaths related to radiation exposure. IMRT has revealed advantages on dose constraint of lungs and heart compared with 3DCRT (25-27). Wang et al. reported total lung V20, V30 reduced approximately from $31 \%, 16 \%$ (3DCRT) to $22 \%, 13 \%$ (IMRT); the heart V30, V40 from 29\%, 21\% (3DCRT) to 28\%, 20\% (IMRT) (25). Data from Memorial Sloan-Kettering Cancer Center also supported IMRT for significant reduction in heart mean dose from 28.2 to 22.9 Gy and V30 from $61.0 \%$ to $24.8 \%$. The Conformality index was also better for IMRT (1.30 vs. 1.56 for 3DCRT) (27). The largest cohort comparing long term survival of 3DCRT and IMRT was from MD Anderson Cancer Center, which reported 676 esophageal cancer patients, 413 were treated by 3DCRT and 263 were treated by IMRT. IMRT significantly associated with prolonged overall survival (median overall survival, 43.2 months for IMRT vs. 25.2 months for 3DCRT, $\mathrm{P}<0.001)$ and decreased locoregional recurrence $(\mathrm{P}=0.0038)$. Although cancer specific death did not differ between groups, the cardiac death was lowered in IMRT group by competing risk analysis $(\mathrm{P}=0.049)(28)$. These trials indicated that the improvement of dosimetric parameters could result into survival benefits.

\section{Volumetric modulated arc radiotherapy (VMAT)}

The disadvantage of IMRT is known as the higher number of MU and longer treatment time (25). VMAT offers possibility in terms of reduction in MU as well as in reducing the treatment time compared with IMRT. The high delivery speed may decrease the risk of intrafractional positional shifts of the patients. Also, it leads to a reduction of doses to normal tissues and improves the homogeneity at various levels (29). Although some studies pointed out that VMAT appeared to spare the lungs from moderate dose irradiation $\left(\mathrm{V}_{20}, \mathrm{~V}_{30}\right)$ at the expense of increasing the low dose irradiation volume $\left(\mathrm{V}_{5}, \mathrm{~V}_{10}\right)(30,31)$, it still indicated equivalent or even better conformity, homogeneity and normal tissue sparing $(31,32)$.

\section{Proton beam therapy (PBT)}

PBT has a physical properties of Bragg peak, which can reduce the radiation dose of surrounding normal tissues significantly (33). Recent studies have revealed that PBT decreased dose to various volumes of the heart and lungs in comparison to the IMRT, especially in cardiac parameters, which indicated decreased risk of cardiac damage and might become one of the primary methods of esophageal cancer treatment $(34,35)$.

PBT is increasingly being taken into consideration in recent years (Table 2). MD Anderson Cancer Center reported the first group of esophageal cancer patients treated by PBT. Sixty-two patients were enrolled and a subset of $29(46.8 \%)$ patients received preoperative concurrent chemotherapy and PBT (median dose 50.4 Gy). pCR $(28 \%)$ and near complete response rates were $50 \%$. The 3-year overall survival, relapse free survival, distant metastasis free survival and locoregional control rates were $51.7 \%, 40.5 \%, 66.7 \%$ and $56.5 \%$, respectively. Postoperative complications for pulmonary, cardiac, gastrointestinal, or wound infections were each less than $10 \%$, which manifested relatively lower morbidities compared with conformal radiotherapy (36). Since the proton beam is known as the sharp drop off beyond the Bragg peak, intrafractional and interfractional bias should be noticed to avoid insufficient dose of target volume. Routine staging procedures such as endoscopy, ultrasonic endoscopy, bronchoscopy, chest/abdomen CT, PET-CT are recommended. Precise simulation (4D-CT and active breath control) could be practiced to consider respiratory movement.

\section{Radiation dosage}

\section{Conventional radiation}

Radiation dose for definitive chemoradiotherapy (50-50.4 Gy) was based on RTOG 8501 and INT 0123 (RTOG 9405) trial. In RTOG 8501, concurrent chemoradiotherapy with 50.4 Gy outperformed radiation alone group in median survival (14 vs. 9 months) and 5 -year survival $(27 \%$ vs. $0 \%, \mathrm{P}<0.0001)$ (38). INT 0123 trial compared different radiation dose for concurrent chemoradiotherapy (64.8 vs. 50.4 Gy). The higher dose group did not reveal survival benefits compared with lower dose group (median survival 13 vs. 18 months, 2-year survival $31 \%$ vs. $40 \%$ ). Locoregional failure or locoregional disease persistence were $56 \%$ and $52 \%$ in higher dose and lower dose group respectively. However, majority of death patients in high dose group did not receive greater than 50.4 Gy dose, and the lower dose of 5-FU cause damage to survival (39). In addition, these evidences were established decades ago, two-dimensional radiation and large margin may cause injury to normal tissues due to lack of proper protection, advanced techniques and new 
chemotherapy regimens largely decrease toxicities and improve compliance. Several studies have demonstrated that radiation dose more than 50 Gy could produce better outcomes. In a Japanese trial, standard dose (54 Gy) and high dose (63 Gy) were given to stage II-III esophageal cancer patients respectively. All the patients received 5 -FU based chemotherapy. High dose group significantly improved 2-year locoregional control rates $(69 \%$ vs. $32 \%$, $\mathrm{P}<0.01), 2$-year progression free survival rates $(47 \% v s$. $20 \%, P=0.01)$, median overall survival was longer but not significant (28vs. 18 months, $\mathrm{P}=0.26$ ), so as complete response rates $(53 \%$ vs. $39 \%, \mathrm{P}=0.14)$. Treatment mortality and late toxicities were not different between groups (40). What should be noticed is that in-field failure rates was significantly higher in standard dose group $(41 \% v s .16 \%$, $\mathrm{P}<0.01$ ), which indicated insufficient dose to the target volume. Zhang et al. (41) also concluded that radiation dose greater than 51 Gy could result in better 3-year local control rate $(36 \%$ vs. $19 \%, \mathrm{P}=0.011)$, disease free survival rate $(25 \%$ vs. $10 \%, \mathrm{P}=0.004)$, overall survival rate $(13 \%$ vs. $3 \%, \mathrm{P}=0.054)$, and clinical complete response (cCR) rate (46\% vs. 23\%, $\mathrm{P}=0.048$ ). Moreover, high dose cause less tumor persistence $(\mathrm{P}=0.02)$ and a trend towards improved distant metastasis free survival $(72 \%$ vs. $59 \%, \mathrm{P}=0.12)$. A recent study claimed that radiation dose more than $60 \mathrm{~Gy}$ had better locoregional control, progression free survival and overall survival, and most of the patients were squamous cell carcinoma (42).

\section{Simultaneous integrated boost (SIB)}

The most common failure pattern of low dose radiation lies in primary tumor (gross target volume, GTV). In a study from the MD Anderson Cancer Center, four-dimensional CT simulation, PET-CT fusion scan, endoscopic ultrasonography and diagnostic CT image were used to ensure the accuracy of GTV delineation. Patients were treated with 50.4 Gy radiation with conventional fraction and concurrent chemotherapy. About 50\% experienced local failure, among these patients, $90 \%$ were in GTV (72\% only in GTV), $23 \%$ in clinical target volume (CTV), and $12 \%$ in planning target volume (PTV). The median overall survival for patients who had GTV failure significantly shorter than who did not (23.3 vs. 31.6 months, $\mathrm{P}=0.0009)$ (43).

SIB aimed to increase radiation dose to GTV and keep lower dose to subclinical areas without sequential boost, which could avoid prolonged treatment duration and obtain more exact dose. A study investigated dosimetry parameters 
of SIB for esophageal carcinoma (44). By using the intensity modulated radiation therapy technique, SIB with boost of 64.8 Gy in 28 fractions to GTV resulted mean GTV dose of $66.9 \mathrm{~Gy}$, whereas conventional dose of $50.4 \mathrm{~Gy}$ in 28 fractions had a mean GTV dose of 52 Gy. Mean lung dose was similar between two groups (7.4 Gy in SIB group vs. $7.7 \mathrm{~Gy}$ in conventional group, $\mathrm{P}=0.06$ ), as well as lung $\mathrm{V}_{20}(13 \%$ in SIB group vs. $12 \%$ in conventional group, $\mathrm{P}=0.06)$. Mean dose were also comparable to heart and liver. Another study also confirmed the feasibility of SIB (45), which significantly improved tumor control probability (TCP) and the increase of normal tissue complication probability (NTCP) were negligible.

Phase I/II trial of SIB in esophageal cancer has been conducted in several centers. Yu et al. reported a phase II

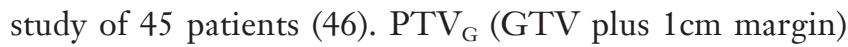
was given 63 Gy in 28 fractions and $\mathrm{PTV}_{\mathrm{C}}(\mathrm{CTV}$ plus $1 \mathrm{~cm}$ margin) was given 50.4 Gy in 28 fractions. CTV involved elective node irradiation (ENI). Concurrent chemotherapy with cisplatin and fluorouracil were administered. The 3 -year progression free survival and overall survival rates were $40.7 \%$ and $42.2 \%$, respectively. Locoregional control rates were $83.3 \%$ at 1 year and $67.5 \%$ at 3 years. No grade 4-5 toxicity was observed. Based on this trial, they added a PTV $_{\text {GR }}$, which included part of GTV that standard uptake value (SUV) more than $50 \%$ of the $\mathrm{SUV}_{\mathrm{MAX}}$ area based on PET-CT scan. No prophylactic irradiation was given except cervical or upper thoracic cancer. Dose escalation achieved Level 4 (50 Gy in $\mathrm{PTV}_{\mathrm{C}}, 62.5$ Gy in $\mathrm{PTV}_{\mathrm{G}}$, $70 \mathrm{~Gy}$ in $\mathrm{PTV}_{\mathrm{GR}}$, all in 25 fractions). No dose limited toxicity was observed. One-year overall survival and local control rates were $69.2 \%$ and $77.4 \%$ respectively (47). Chen et al. used SIB with 54 Gy at $1.8 \mathrm{~Gy} /$ fraction to subclinical disease and 66 Gy at $2.2 \mathrm{~Gy} /$ fraction to GTV. The 1- and 2-year locoregional control, disease-free survival and overall survival rates were $87.6 \%$ and $78.6 \%, 75.6 \%$ and $64.4 \%$, $86.7 \%$ and $72.7 \%$, respectively (48). Welsh et al. conducted a phase I/II trial. In this study, unresectable esophageal cancer patients were given chemoradiation with an SIB of 58.8-63 Gy to the GTV and 50.4 Gy to the PTV, all in 28 fractions. Compared with standard 50.4 Gy radiation, SIB reduced the local failure rates for patients with positive nodes $(13 \%$ vs. $56 \%, \mathrm{P}=0.04)$, adenocarcinoma ( $26 \%$ vs. $59 \%, \mathrm{P}=0.02)$, and stage III-IV disease (29\% vs. $55 \%$, $\mathrm{P}=0.04$ ) (49). The prospect of SIB seems to be promising, with the purpose of enhancing locoregional control or even harvesting survival benefits. Further studies and phase III randomized trials are being investigated.

\section{Target volume}

One of the most controversial topic in radiation target volume of esophageal cancer is whether ENI or involved field irradiation (IFI). The evidence of ENI was derived from prophylactic lymph node dissection in Japan. It has revealed three-field lymph node dissection can recognize occult metastasis, and potentially improves survival by eliminating micrometastases and reducing the regional lymph node recurrence rates (50). A study investigated the recurrence pattern of ENI. In this trial, primary tumor and metastatic lymph nodes were given 60 Gy. ENI was delivered to lower cervical, periesophageal, mediastinal and perigastric nodes. Supraclavicular nodes were included for upper thoracic esophageal cancer and celiac nodes were included for lower esophageal cancer. As a result, only one $(1.0 \%)$ patient experienced elective nodal failure without any other site of recurrence (51). Another study defined ENI region as the whole thoracic esophagus (from the supraclavicular fossae to the gastroesophageal junction), radiation was given 50-50.4 Gy with concurrent chemotherapy, after more than 2 years follow up, no patient experienced elective nodal failure without any other site of recurrence (52). ENI was proved to reduce regional lymph node failure, but no evidence of survival benefits was showed (53). The incidence of locoregional failure and persistence of disease in chemoradiotherapy arm of RTOG 8501, which used ENI, was lower than that in the standard dose arm of INT 0123, which omitted ENI (46\% vs. 55\%), but the median survival times and the 2-year overall survival rates were similar between groups (14.1 months, $36 \% v s$. 18.1 months, $40 \%$ ).

As to the comparison of ENI and IFI, Yamashita et al. found that 3-year locoregional control $(44.8 \%$ vs. $55.5 \%, \mathrm{P}=0.039)$, distant control $(53.8 \%$ vs. $69.9 \%$, $\mathrm{P}=0.021)$ and overall survival $(34.8 \%$ vs. $51.6 \%, \mathrm{P}=0.087)$ rates of ENI was inferior to IFI, mainly contribute to toxicities of whole thoracic esophagus irradiation $(16 \%$ vs. $8 \%, \mathrm{P}=0.047)$. IFI did not increase the risk of initially uninvolved or isolated nodal failures $(27.5 \%$ in ENI $v s$. $13.4 \%$ in IFI) (54). According to recurrence pattern, out-of-field failure was not the major failure pattern and even did not cause significant impact on survival $(55,56)$. Since in-field recurrence and distant metastases were the most important failure pattern and appears earlier than out-of-field recurrence, it was suggested that IFI should be an alternative way. Exceptionally, cervical and supraclavicular lymph nodes are the common recurrent 
sites after surgery or chemoradiotherapy, especially for upper thoracic or cervical esophageal cancer, and salvage surgery may be more difficult for these areas, ENI of lower cervical and supraclavicular lymph nodes should be considered for upper thoracic or cervical esophageal cancer patients.

\section{Radiotherapy in elderly patients}

Increasing numbers of elderly patients with comorbidities and impaired performance status were a problem cannot be neglected. Definitive chemoradiotherapy is usually the treatment option for elderly esophageal cancer patients. Tougeron et al. reported 109 non-metastatic esophageal patients older than 70 years treated by cisplatinbased definitive chemoradiotherapy, radiation dose was 50-55 Gy. cCR rates was $57.8 \%$ and 2-year survival was $35.5 \%$. However, greater than grade 3 adverse events were observed in $23.8 \%$ patients. Chemotherapy dose reduction, chemotherapy delays more than 1 week, and treatment discontinuation were observed in $30.3 \%, 41.3 \%$, and $15.6 \%$ patients, respectively. Multivariate analysis showed that apart from cCR, radiation dose $\geq 80 \%$ and Charlson score $\leq 2$ were identified as independent prognostic factors of overall survival (57). Another study compared the treatment and toxicities of elderly (age $\geq 71$ ) and nonelderly (age $\leq 70$ ) patients. Both group received fluorouracil and cisplatin chemotherapy 60 Gy of radiation. Although cCR rates were almost identical (63.6\% for elderly vs. 63.4\% for nonelderly), recurrence rates after cCR was higher in elderly (47.6\% vs. $33.7 \%, \mathrm{P}=0.32$ ), median survival was much lower (14.7 vs. 35.1 months, $\mathrm{P}=0.01$ ), and discontinuation at the end of chemoradiotherapy was much frequent in elderly (57.6\% vs. $17.3 \%, \mathrm{P}=0.01$ ) (58).

It should be noticed that elderly patients usually be excluded by clinical trials owing to insufficient organ function or other comorbidities. Retrospective analyses indicated that although promising response of chemoradiotherapy could been seen, elderly patients more likely suffered from adverse events, which harmed treatment compliance and reduce efficacy. Radiation oncologists are trying to take measures to reduce toxicities, such as using IFI instead of ENI, or finding more tolerable chemotherapy regimen, both revealed acceptable results $(59,60)$.

\section{Acknowledgements}

None.

\section{Footnote}

Conflicts of Interest: The authors have no conflicts of interest to declare.

\section{References}

1. Torre LA, Bray F, Siegel RL, et al. Global cancer statistics, 2012. CA Cancer J Clin 2015;65:87-108.

2. Sjoquist KM, Burmeister BH, Smithers BM, et al. Survival after neoadjuvant chemotherapy or chemoradiotherapy for resectable oesophageal carcinoma: an updated metaanalysis. Lancet Oncol 2011;12:681-92.

3. Kranzfelder M, Schuster T, Geinitz H, et al. Meta-analysis of neoadjuvant treatment modalities and definitive nonsurgical therapy for oesophageal squamous cell cancer. Br J Surg 2011;98:768-83.

4. van Hagen P, Hulshof MC, van Lanschot JJ, et al. Preoperative chemoradiotherapy for esophageal or junctional cancer. N Engl J Med 2012;366:2074-84.

5. Shapiro J, van Lanschot JJB, Hulshof MCCM, et al. Neoadjuvant chemoradiotherapy plus surgery versus surgery alone for oesophageal or junctional cancer (CROSS): long-term results of a randomised controlled trial. Lancet Oncol 2015;16:1090-8.

6. Cunningham D, Allum WH, Stenning SP, et al. Perioperative chemotherapy versus surgery alone for resectable gastroesophageal cancer. $\mathrm{N}$ Engl J Med 2006;355:11-20.

7. Klevebro F, Alexandersson von Dobeln G, Wang N, et al. A randomized clinical trial of neoadjuvant chemotherapy versus neoadjuvant chemoradiotherapy for cancer of the oesophagus or gastro-oesophageal junction. Ann Oncol 2016;27:660-7.

8. Clavien PA, Barkun J, de Oliveira ML, et al. The ClavienDindo classification of surgical complications: five-year experience. Ann Surg 2009;250:187-96.

9. Kumagai K, Rouvelas I, Tsai JA, et al. Meta-analysis of postoperative morbidity and perioperative mortality in patients receiving neoadjuvant chemotherapy or chemoradiotherapy for resectable oesophageal and gastrooesophageal junctional cancers. Br J Surg 2014;101:321-38.

10. Al-Sukhni E, Gabriel E, Attwood K, et al. No Survival Difference with Neoadjuvant Chemoradiotherapy Compared with Chemotherapy in Resectable Esophageal and Gastroesophageal Junction Adenocarcinoma: Results from the National Cancer Data Base. J Am Coll Surg 2016;223:784-92.e1. 
11. Luu TD, Gaur P, Force SD, et al. Neoadjuvant chemoradiation versus chemotherapy for patients undergoing esophagectomy for esophageal cancer. Ann Thorac Surg 2008;85:1217-23; discussion 1223-4.

12. Stahl M, Walz MK, Stuschke M, et al. Phase III comparison of preoperative chemotherapy compared with chemoradiotherapy in patients with locally advanced adenocarcinoma of the esophagogastric junction. J Clin Oncol 2009;27:851-6.

13. Swisher SG, Hofstetter W, Komaki R, et al. Improved long-term outcome with chemoradiotherapy strategies in esophageal cancer. Ann Thorac Surg 2010;90:892-8; discussion 898-9.

14. Morgan MA, Lewis WG, Crosby TD, et al. Prospective cohort comparison of neoadjuvant chemoradiotherapy versus chemotherapy in patients with oesophageal cancer. Br J Surg 2007;94:1509-14.

15. Burmeister BH, Thomas JM, Burmeister EA, et al. Is concurrent radiation therapy required in patients receiving preoperative chemotherapy for adenocarcinoma of the oesophagus? A randomised phase II trial. Eur J Cancer 2011;47:354-60.

16. Favi F, Bollschweiler E, Berlth F, et al. Neoadjuvant chemotherapy or chemoradiation for patients with advanced adenocarcinoma of the oesophagus? A propensity score-matched study. Eur J Surg Oncol 2017;43:1572-80.

17. Li QQ, Liu MZ, Hu YH, et al. Definitive concomitant chemoradiotherapy with docetaxel and cisplatin in squamous esophageal carcinoma. Dis Esophagus 2010;23:253-9.

18. Honing J, Smit JK, Muijs CT, et al. A comparison of carboplatin and paclitaxel with cisplatinum and 5 -fluorouracil in definitive chemoradiation in esophageal cancer patients. Ann Oncol 2014;25:638-43.

19. Bedenne L, Michel P, Bouche O, et al. Chemoradiation followed by surgery compared with chemoradiation alone in squamous cancer of the esophagus: FFCD 9102. J Clin Oncol 2007;25:1160-8.

20. Stahl M, Stuschke M, Lehmann N, et al. Chemoradiation with and without surgery in patients with locally advanced squamous cell carcinoma of the esophagus. J Clin Oncol 2005;23:2310-7.

21. Piessen G, Messager M, Mirabel X, et al. Is there a role for surgery for patients with a complete clinical response after chemoradiation for esophageal cancer? An intentionto-treat case-control study. Ann Surg 2013;258:793-9; discussion 799-800.

22. Markar S, Gronnier C, Duhamel A, et al. Salvage
Surgery After Chemoradiotherapy in the Management of Esophageal Cancer: Is It a Viable Therapeutic Option? J Clin Oncol 2015;33:3866-73.

23. Marks JL, Hofstetter W, Correa AM, et al. Salvage esophagectomy after failed definitive chemoradiation for esophageal adenocarcinoma. Ann Thorac Surg 2012;94:1126-32; discussion 1132-3.

24. Xi M, Xu C, Liao Z, et al. The impact of histology on recurrence patterns in esophageal cancer treated with definitive chemoradiotherapy. Radiother Oncol 2017;124:318-24..

25. Wang D, Yang Y, Zhu J, et al. 3D-conformal RT, fixed-field IMRT and RapidArc, which one is better for esophageal carcinoma treated with elective nodal irradiation. Technol Cancer Res Treat 2011;10:487-94.

26. Wu VW, Sham JS, Kwong DL. Inverse planning in three-dimensional conformal and intensity-modulated radiotherapy of mid-thoracic oesophageal cancer. Br J Radiol 2004;77:568-72.

27. Kole TP, Aghayere O, Kwah J, et al. Comparison of heart and coronary artery doses associated with intensitymodulated radiotherapy versus three-dimensional conformal radiotherapy for distal esophageal cancer. Int J Radiat Oncol Biol Phys 2012;83:1580-6.

28. Lin SH, Wang L, Myles B, et al. Propensity score-based comparison of long-term outcomes with 3-dimensional conformal radiotherapy vs intensity-modulated radiotherapy for esophageal cancer. Int J Radiat Oncol Biol Phys 2012;84:1078-85.

29. Vivekanandan N, Sriram P, Kumar SA, et al. Volumetric modulated arc radiotherapy for esophageal cancer. Med Dosim 2012;37:108-13.

30. Lin CY, Huang WY, Jen YM, et al. Dosimetric and efficiency comparison of high-dose radiotherapy for esophageal cancer: volumetric modulated arc therapy versus fixed-field intensity-modulated radiotherapy. Dis Esophagus 2014;27:585-90.

31. Zhang WZ, Zhai TT, Lu JY, et al. Volumetric modulated arc therapy vs. c-IMRT for the treatment of upper thoracic esophageal cancer. PLoS One 2015;10:e0121385.

32. Yin L, Wu H, Gong J, et al. Volumetric-modulated arc therapy vs. c-IMRT in esophageal cancer: a treatment planning comparison. World J Gastroenterol 2012;18:5266-75.

33. Terasawa T, Dvorak T, Ip S, et al. Systematic review: charged-particle radiation therapy for cancer. Ann Intern Med 2009;151:556-65.

34. Ling TC, Slater JM, Nookala P, et al. Analysis of 
Intensity-Modulated Radiation Therapy (IMRT), Proton and 3D Conformal Radiotherapy (3D-CRT) for Reducing Perioperative Cardiopulmonary Complications in Esophageal Cancer Patients. Cancers (Basel) 2014;6:2356-68.

35. Takada A, Nakamura T, Takayama K, et al. Preliminary treatment results of proton beam therapy with chemoradiotherapy for stage I-III esophageal cancer. Cancer Med 2016;5:506-15.

36. Lin SH, Komaki R, Liao Z, et al. Proton beam therapy and concurrent chemotherapy for esophageal cancer. Int J Radiat Oncol Biol Phys 2012;83:e345-51.

37. Ishikawa H, Hashimoto T, Moriwaki T, et al. Proton beam therapy combined with concurrent chemotherapy for esophageal cancer. Anticancer Res 2015;35:1757-62.

38. al-Sarraf M, Martz K, Herskovic A, et al. Progress report of combined chemoradiotherapy versus radiotherapy alone in patients with esophageal cancer: an intergroup study. J Clin Oncol 1997;15:277-84.

39. Minsky BD, Pajak TF, Ginsberg RJ, et al. INT 0123 (Radiation Therapy Oncology Group 94-05) phase III trial of combined-modality therapy for esophageal cancer: high-dose versus standard-dose radiation therapy. J Clin Oncol 2002;20:1167-74.

40. Suh YG, Lee IJ, Koom WS, et al. High-dose versus standard-dose radiotherapy with concurrent chemotherapy in stages II-III esophageal cancer. Jpn J Clin Oncol 2014;44:534-40.

41. Zhang Z, Liao Z, Jin J, et al. Dose-response relationship in locoregional control for patients with stage II-III esophageal cancer treated with concurrent chemotherapy and radiotherapy. Int J Radiat Oncol Biol Phys 2005;61:656-64.

42. Kim HJ, Suh YG, Lee YC, et al. Dose-Response Relationship between Radiation Dose and Loco-regional Control in Patients with Stage II-III Esophageal Cancer Treated with Definitive Chemoradiotherapy. Cancer Res Treat 2017;49:669-77.

43. Welsh J, Settle SH, Amini A, et al. Failure patterns in patients with esophageal cancer treated with definitive chemoradiation. Cancer 2012;118:2632-40.

44. Welsh J, Palmer MB, Ajani JA, et al. Esophageal cancer dose escalation using a simultaneous integrated boost technique. Int J Radiat Oncol Biol Phys 2012;82:468-74.

45. Warren S, Partridge M, Carrington R, et al. Radiobiological determination of dose escalation and normal tissue toxicity in definitive chemoradiation therapy for esophageal cancer. Int J Radiat Oncol Biol Phys
2014;90:423-9.

46. Yu WW, Zhu ZF, Fu XL, et al. Simultaneous integrated boost intensity-modulated radiotherapy in esophageal carcinoma: early results of a phase II study. Strahlenther Onkol 2014;190:979-86.

47. Yu W, Cai XW, Liu Q, et al. Safety of dose escalation by simultaneous integrated boosting radiation dose within the primary tumor guided by (18)FDG-PET/CT for esophageal cancer. Radiother Oncol 2015;114:195-200.

48. Chen J, Guo H, Zhai T, et al. Radiation dose escalation by simultaneous modulated accelerated radiotherapy combined with chemotherapy for esophageal cancer: a phase II study. Oncotarget 2016;7:22711-9.

49. Welsh JW, Seyedin SN, Allen PK, et al. Local Control and Toxicity of a Simultaneous Integrated Boost for Dose Escalation in Locally Advanced Esophageal Cancer: Interim Results from a Prospective Phase I/II Trial. J Thorac Oncol 2017;12:375-82.

50. Fujita H, Kakegawa T, Yamana $\mathrm{H}$, et al. Mortality and morbidity rates, postoperative course, quality of life, and prognosis after extended radical lymphadenectomy for esophageal cancer. Comparison of three-field lymphadenectomy with two-field lymphadenectomy. Ann Surg 1995;222:654-62.

51. Onozawa $M$, Nihei K, Ishikura $S$, et al. Elective nodal irradiation (ENI) in definitive chemoradiotherapy (CRT) for squamous cell carcinoma of the thoracic esophagus. Radiother Oncol 2009;92:266-9.

52. Yamashita H, Okuma K, Wakui R, et al. Details of recurrence sites after elective nodal irradiation (ENI) using 3D-conformal radiotherapy (3D-CRT) combined with chemotherapy for thoracic esophageal squamous cell carcinoma--a retrospective analysis. Radiother Oncol 2011;98:255-60.

53. Hsu FM, Lee JM, Huang PM, et al. Retrospective analysis of outcome differences in preoperative concurrent chemoradiation with or without elective nodal irradiation for esophageal squamous cell carcinoma. Int J Radiat Oncol Biol Phys 2011;81:e593-9.

54. Yamashita H, Takenaka R, Omori M, et al. Involvedfield radiotherapy (IFRT) versus elective nodal irradiation (ENI) in combination with concurrent chemotherapy for 239 esophageal cancers: a single institutional retrospective study. Radiat Oncol 2015;10:171.

55. Zhang X, Li M, Meng X, et al. Involved-field irradiation in definitive chemoradiotherapy for locally advanced esophageal squamous cell carcinoma. Radiat Oncol 2014;9:64. 
56. Zhang X, Yu J, Li M, et al. Details of out-field regional recurrence after involved-field irradiation with concurrent chemotherapy for locally advanced esophageal squamous cell carcinoma. Onco Targets Ther 2016;9:3049-57.

57. Tougeron D, Di Fiore F, Thureau S, et al. Safety and outcome of definitive chemoradiotherapy in elderly patients with oesophageal cancer. Br J Cancer 2008;99:1586-92.

58. Takeuchi S, Ohtsu A, Doi T, et al. A retrospective study of definitive chemoradiotherapy for elderly patients with esophageal cancer. Am J Clin Oncol 2007;30:607-11.

Cite this article as: Deng W, Lin SH. Advances in radiotherapy for esophageal cancer. Ann Transl Med 2018;6(4):79. doi: 10.21037/atm.2017.11.28
59. Jing $\mathrm{W}$, Zhu $\mathrm{H}$, Guo $\mathrm{H}$, et al. Feasibility of Elective Nodal Irradiation (ENI) and Involved Field Irradiation (IFI) in Radiotherapy for the Elderly Patients (Aged $>/=$ 70 Years) with Esophageal Squamous Cell Cancer: A Retrospective Analysis from a Single Institute. PLoS One 2015;10:e0143007.

60. Chen Y, Zhao K, Zhu Z, et al. Preliminary Results of a Phase 2 Study of Definitive Chemoradiation Therapy Using S-1 for Esophageal Squamous Cell Carcinoma Patients. Int J Radiat Oncol Biol Phys 2016;96:S30-S1. 